PUPT-2424

\title{
Exact ABJM Partition Function from TBA
}

\author{
Pavel Putrov* and Masahito Yamazaki^ \\ * Département de Physique Théorique et Section de Mathématiques, \\ Université de Genève, Genève, CH-1211, Switzerland \\ - Princeton Center for Theoretical Science, Princeton University, NJ 08544, USA
}

\begin{abstract}
We report on the exact computation of the $S^{3}$ partition function of $U(N)_{k} \times U(N)_{-k}$ ABJM theory for $k=1, N=1, \ldots, 19$. The result is a polynomial in $\pi^{-1}$ with rational coefficients. As an application of our results we numerically determine the coefficient of the membrane 1-instanton correction to the partition function.
\end{abstract}




\section{Introduction and Summary}

It has recently been discovered that the partition function of a Chern-Simons-matter (CSM) theory with $\mathcal{N} \geq 2$ supersymmetry on a three-dimensional sphere reduces to a matrix integral [1, 2, 3. These matrix integrals are powerful quantitative tools to analyze CSM theories, and has lead to a number of important results, including the successful derivation of the $N^{3 / 2}$ behavior [4] and various precise checks of the $\mathrm{AdS}_{4} / \mathrm{CFT}_{3}$ correspondence (see [5, 6, 7] and subsequent works).

In this paper we study the CSM theory with the highest amount of supersymmetry $(\mathcal{N} \geq 6)$, namely the ABJM theory [8]. It has gauge group $U(N)_{k} \times U(N)_{-k}$, where $k$ is the level of the Chern-Simons term. Since ABJM theory is the worldvolume theory of multiple M2-branes, it is natural to ask if we could extract any useful data about M-theory from the three-sphere partition function of the ABJM theory.

In M-theory we have non-perturbative corrections from membrane instantons. This is reflected in the three-sphere partition function as an expansion of the terms of order $e^{-\sqrt{N / k}}[9$. However, this expansion is not directly captured in most of the previous analysis of the three-sphere partition function, where we take the t' Hooft limit $N, k$ large with $N / k$ kept finite. Instead we need to take the M-theory limit, with $N$ large and $k$ kept finite. The leading $N$ contribution in this limit is determined by [7] and the all order $1 / N$ expansion in [10]. Moreover the paper [10] discuss the non-perturtbative instanton correction in an expansion around $k=0$. However the for the most interesting case of $k$ finite, the general results on the non-perturbative corrections are still lacking. To answer this question it will be of great help to systematically compute the behavior of the three-sphere partition function for finite $N$ and $k$.

In this brief note we report on the exact computation of $S^{3}$ partition function $Z(N)$ of the $k=1$ $(\mathcal{N}=8)$ ABJM theory for $N=1, \ldots, 19$, based on the Fermi gas approach of [10] and the TBA-like equations of [11, 12, 1$]$

\footnotetext{
${ }^{1}$ See 13 for exact computation for $N=2$ and general $k$, and 14 for numerical calculations.
} 
Our results are given as follows:

$$
\begin{aligned}
Z(1) & =\frac{1}{4}, \quad Z(2)=\frac{1}{16 \pi}, \quad Z(3)=\frac{-3+\pi}{64 \pi}, \quad Z(4)=\frac{-\pi^{2}+10}{1024 \pi^{2}}, \quad Z(5)=\frac{26+20 \pi-9 \pi^{2}}{4096 \pi^{2}} \\
Z(6) & =\frac{78-121 \pi^{2}+36 \pi^{3}}{147456 \pi^{3}}, \quad Z(7)=\frac{-126+174 \pi+193 \pi^{2}-75 \pi^{3}}{196608 \pi^{3}}, \\
Z(8) & =\frac{876-4148 \pi^{2}-2016 \pi^{3}+1053 \pi^{4}}{18874368 \pi^{4}}, \quad Z(9)=\frac{4140+8880 \pi-15348 \pi^{2}-13480 \pi^{3}+5517 \pi^{4}}{75497472 \pi^{4}} \\
Z(10) & =\frac{16860-136700 \pi^{2}+190800 \pi^{3}+207413 \pi^{4}-81000 \pi^{5}}{7549747200 \pi^{5}}, \\
Z(11) & =\frac{-122580+381900 \pi+837300 \pi^{2}-1289300 \pi^{3}-1091439 \pi^{4}+447525 \pi^{5}}{30198988800 \pi^{5}}, \\
Z(12) & =\frac{626760-8856300 \pi^{2}-18446400 \pi^{3}+35287138 \pi^{4}+30204000 \pi^{5}-12504375 \pi^{6}}{4348654387200 \pi^{6}}, \\
Z(13) & =\frac{1563480+6714000 \pi-17252100 \pi^{2}-40746000 \pi^{3}+49141894 \pi^{4}+45780780 \pi^{5}-18083925 \pi^{6}}{5798205849600 \pi^{6}} \\
Z(14) & =\left(21382200-421152060 \pi^{2}+1918350000 \pi^{3}+2614227910 \pi^{4}-\right. \\
& \left.-5654854800 \pi^{5}-3965159223 \pi^{6}+1732468500 \pi^{7}\right) /\left(3409345039564800 \pi^{7}\right) \\
Z(15) & =\left(-222059880+1271579400 \pi+3613033620 \pi^{2}-12266517900 \pi^{3}-17757814914 \pi^{4}+\right. \\
& \left.+28941378130 \pi^{5}+21727092861 \pi^{6}-9162734175 \pi^{7}\right) /\left(13637380158259200 \pi^{7}\right) \\
Z(16) & =\left(288454320-8196414240 \pi^{2}-54540622080 \pi^{3}+83379537976 \pi^{4}+337956998400 \pi^{5}-310977507352 \pi^{6}-\right. \\
& \left.-354450849984 \pi^{7}+132764935275 \pi^{8}\right) /\left(872792330128588800 \pi^{8}\right) \\
Z(17) & =\left(3171011760+23555952000 \pi-71723746080 \pi^{2}-333199608000 \pi^{3}+542885550648 \pi^{4}+1355261623520 \pi^{5}-\right. \\
& \left.-1384280129304 \pi^{6}-1337978574000 \pi^{7}+518021476875 \pi^{8}\right) /\left(3491169320514355200 \pi^{8}\right) \\
Z(18) & =\left(4970745360-180631896480 \pi^{2}+2270514395520 \pi^{3}+2444801550408 \pi^{4}-\right. \\
& -18251132155200 \pi^{5}-13590443330584 \pi^{6}+35949047139936 \pi^{7}+ \\
& \left.+20671882502409 \pi^{8}-9607077219600 \pi^{9}\right) /\left(377046286615550361600 \pi^{9}\right) \\
Z(19) & =\left(-2636096400+24895105200 \pi+79219113120 \pi^{2}-487774106400 \pi^{3}-\right. \\
& -852843285000 \pi^{4}+3053792290360 \pi^{5}+3630439618136 \pi^{6}-6122444513560 \pi^{7}- \\
& \left.-4288974330849 \pi^{8}+1840384320075 \pi^{9}\right) /\left(55858709128229683200 \pi^{9}\right) \\
&
\end{aligned}
$$

Section 2 of this paper is devoted to the derivation of this result. Similar methods could be applied to $k>1$. It would be interesting to find an analytic expression for general $k$ and $N$.

The knowledge of the exact values of $Z(N)$ in this paper allows one to perform various numerical tests with high precision. As an example, we compute in Section 3 the coefficient of the membrane 1-instanton contribution to the partition function (see (30p).

Note: During the preparation of this manuscript we received a paper [16], which has substantial overlap with our paper. The paper contains the exact results up to $N=9$, which is consistent with ours. 


\section{Derivation}

Let us consider the grand canonical partition function

$$
\Xi(z)=1+\sum_{N \geq 1} Z(N) z^{N}
$$

As is shown in [10, this is given by a Fredholm determinant

$$
\Xi(z)=\operatorname{Det}\left(1+\frac{z \hat{K}}{4 \pi}\right)
$$

with $\hat{K}$ defined by an integral kernel

$$
K(x, y):=\langle x|\hat{K}| y\rangle=\frac{e^{-u(x)-u(y)}}{\cosh \left(\frac{x-y}{2}\right)},
$$

and

$$
u(x)=\frac{1}{2} \log \left(2 \cosh \frac{k x}{2}\right) .
$$

In practice, it is useful to use the following relation:

$$
\Xi(z)=\exp \left(\operatorname{Tr} \log \left(1+\frac{z \hat{K}}{4 \pi}\right)\right)=\exp \left(-\sum_{\ell} Z_{\ell} \frac{(-z)^{\ell}}{\ell}\right),
$$

with

$$
Z_{\ell}=\frac{1}{(4 \pi)^{\ell}} \operatorname{Tr}\left(\hat{K}^{\ell}\right)=\frac{1}{(4 \pi)^{\ell}} \int d x_{1} \ldots d x_{\ell} K\left(x_{1}, x_{2}\right) K\left(x_{2}, x_{3}\right) \ldots K\left(x_{\ell-1}, x_{\ell}\right) K\left(x_{\ell}, x_{1}\right) .
$$

The problem thus reduces to the computation of $Z_{\ell}$.

Let us define the kernel for the operator $\hat{K}\left(I-\lambda^{2} \hat{K}^{2}\right)^{-1}$ by $R_{+}(x, y)$ and for $\lambda \hat{K}^{2}\left(I-\lambda^{2} \hat{K}^{2}\right)^{-1}$ by $R_{-}(x, y)$, respectively. We also denote $R_{+}(x):=R_{+}(x, x), R_{-}(x):=R_{-}(x, x)$. As is clear from the definition, the integral of $R_{ \pm}(x)$ gives $Z_{\ell}$ :

$$
\frac{1}{4 \pi} \int d x R_{+}(x)=\sum_{n \geq 0}(4 \pi \lambda)^{2 n} Z_{2 n+1}, \quad \frac{1}{4 \pi} \int d x R_{-}(x)=\sum_{n \geq 0}(4 \pi \lambda)^{2 n+1} Z_{2 n+2} .
$$

Let us further define $\epsilon(\theta), \eta(\theta)$ by

$$
e^{-\epsilon(\theta)}=R_{+}(\theta), \quad \eta(\theta)=2 \lambda \int_{-\infty}^{\infty} \frac{e^{-\epsilon\left(\theta^{\prime}\right)}}{\cosh \left(\theta-\theta^{\prime}\right)} d \theta^{\prime} .
$$

It was conjectured in [11] and later proven in [12] that these functions satisfy the following two TBA-like equations:

$$
\begin{aligned}
& \epsilon(\theta)=2 u(\theta)-\frac{1}{2 \pi} \int_{-\infty}^{\infty} \frac{\log \left(1+\eta^{2}\left(\theta^{\prime}\right)\right)}{\cosh \left(\theta-\theta^{\prime}\right)} d \theta^{\prime} \\
& R_{-}(\theta)=\frac{1}{\pi} R_{+}(\theta) \int_{-\infty}^{\infty} \frac{\arctan \eta\left(\theta^{\prime}\right)}{\cosh ^{2}\left(\theta-\theta^{\prime}\right)} d \theta^{\prime}
\end{aligned}
$$


Let us define

$$
\begin{aligned}
& \epsilon(\theta)=\sum_{n \geq 0} \epsilon_{n}(\theta) \lambda^{2 n}, \quad \eta(\theta)=\sum_{n \geq 0} \eta_{n}(\theta) \lambda^{2 n+1} \\
& R_{+}(\theta)=\sum_{n \geq 0} R_{+, n}(\theta) \lambda^{2 n}, \quad R_{-}(\theta)=\sum_{n \geq 0} R_{-, n}(\theta) \lambda^{2 n+1} .
\end{aligned}
$$

Suppose $\epsilon_{n}(\theta), n=0 \ldots j$ are known. We can then find $\eta_{n}(\theta), n=0 \ldots j$ by performing the integration in (9), and then $\epsilon_{j+1}(\theta)$ from (10). Thus one can solve the TBA-like equations recursively, order by order in $\lambda$, starting from

$$
\epsilon_{0}(\theta)=2 u(\theta)
$$

Once we know $\epsilon_{n}(\theta)$ and $\eta_{n}(\theta)$ for $n=0 \ldots N$ we can find $R_{+, n}(\theta)$ and $R_{-, n}(\theta)$ for $n=0 \ldots N$ and, therefore, $Z_{2 n+1}$ and $Z_{2 n+2}$ for $n=0 \ldots N$ from (8).

Practically, it is useful to make the following change of variables: $e^{\frac{\theta}{2}}=t$. Then the equations $(9,10$ read

$$
\begin{aligned}
& \eta(t)=8 \lambda \int_{0}^{\infty} d s \frac{t^{2} s e^{-\epsilon(s)}}{s^{4}+t^{4}} \\
& \epsilon(t)=\log \left(t^{k}+\frac{1}{t^{k}}\right)-\frac{2}{\pi} \int_{0}^{\infty} d s \frac{t^{2} s \log \left(1+\eta^{2}(s)\right)}{s^{4}+t^{4}}
\end{aligned}
$$

Let us specialize to $k=1$ for simplicity. One can show that the functions $\epsilon_{n}(t), \eta_{n}(t)$ have rather simple structure:

$$
\begin{aligned}
& \epsilon_{n}(t)=\sum_{j=0}^{n-1} G_{j}^{(n)}(t)(\log t)^{j}, n \geq 2, \\
& \eta_{n}(t)=\sum_{j=0}^{n} H_{j}^{(n)}(t)(\log t)^{j}, n \geq 0,
\end{aligned}
$$

where $G_{j}^{(n)}(t)$ are rational functions with poles allowed at the roots of $t^{4}-1$ and $H_{j}^{(n)}(t)$ are rational functions with poles allowed at the roots of $t^{4}+1$. This observation allows easy calculation of the integrals 15 16) order by order in $\lambda$ with the help of the residue theorem and the following formula:

$$
\int_{0}^{\infty} d t C(t)(\log t)^{j}=-\frac{(2 \pi i)^{j}}{j+1} \int_{\gamma} d t C(t) B_{j+1}\left(\frac{\log t}{2 \pi i}\right),
$$

where in the right hand side $\log t$ has a brunch cut from 0 to $+\infty$, the contour $\gamma$ goes from $+\infty$ to 0 below the cut and then to $+\infty$ above the cut, $C(t)$ is a rational function and $B_{j+1}(x)$ is Bernoulli polynomial. 
Using the described procedure we find 2 .

$$
\begin{aligned}
Z_{1} & =\frac{1}{4} \\
Z_{2} & =\frac{-2+\pi}{16 \pi}, \\
Z_{3} & =\frac{\pi-3}{16 \pi}, \\
Z_{4} & =\frac{-4-8 \pi+3 \pi^{2}}{128 \pi^{2}}, \\
Z_{5} & =\frac{10-\pi^{2}}{256 \pi^{2}}, \\
Z_{6} & =\frac{36-2 \pi-3 \pi^{2}}{1536 \pi^{2}}, \\
Z_{7} & =\frac{-42+126 \pi+49 \pi^{2}-27 \pi^{3}}{9216 \pi^{3}}, \\
Z_{8} & =\frac{-96+96 \pi+64 \pi^{2}-27 \pi^{3}}{18432 \pi^{3}}, \\
Z_{9} & =\frac{12-96 \pi-20 \pi^{2}+5 \pi^{4}}{32768 \pi^{4}}, \\
Z_{10} & =\frac{1200-2400 \pi-1400 \pi^{2}+226 \pi^{3}+135 \pi^{4}}{1474560 \pi^{4}}, \\
Z_{11} & =\frac{-660+23100 \pi-12100 \pi^{2}-25300 \pi^{3}-6303 \pi^{4}+4725 \pi^{5}}{29491200 \pi^{5}} \\
Z_{12} & =\frac{-720+3600 \pi+1200 \pi^{2}-2560 \pi^{3}-1536 \pi^{4}+675 \pi^{5}}{7372800 \pi^{5}} \\
Z_{13} & =\frac{4680-561600 \pi+978900 \pi^{2}+655200 \pi^{3}+10114 \pi^{4}-30375 \pi^{6}}{4246732800 \pi^{6}}, \\
Z_{14} & =\frac{141120-1693440 \pi+764400 \pi^{2}+1764000 \pi^{3}+625436 \pi^{4}-162882 \pi^{5}-70875 \pi^{6}}{14863564800 \pi^{6}}, \\
Z_{15} & =\frac{-2520+1076040 \pi-4024860 \pi^{2}-1425900 \pi^{3}+2429714 \pi^{4}+2860522 \pi^{5}+527265 \pi^{6}-509355 \pi^{7}}{55490641920 \pi^{7}}, \\
Z_{16} & =\frac{-40320+1128960 \pi-1599360 \pi^{2}-1646400 \pi^{3}-238336 \pi^{4}+1136128 \pi^{5}+663552 \pi^{6}-297675 \pi^{7}}{52022476800 \pi^{7}}, \\
Z_{17} & =\left(85680-124750080 \pi+931227360 \pi^{2}-303878400 \pi^{3}-1054571336 \pi^{4}-405544384 \pi^{5}+\right. \\
& \left.+45621608 \pi^{6}+19348875 \pi^{8}\right) /\left(53271016243200 \pi^{8}\right), \\
Z_{18} & =\left(80640-5160960 \pi+15617280 \pi^{2}+6397440 \pi^{3}-10554208 \pi^{4}-11079488 \pi^{5}-\right. \\
& \left.-2895216 \pi^{6}+1060922 \pi^{7}+385875 \pi^{8}\right) /\left(1479750451200 \pi^{8}\right), \\
Z_{19} & \left(-287280+1414279440 \pi-20169928800 \pi^{2}+24409032480 \pi^{3}+31396649256 \pi^{4}+1177819272 \pi^{5}-\right. \\
& \left.=19209555560 \pi^{6}-17783325576 \pi^{7}-2533741371 \pi^{8}+3094331625 \pi^{9}\right) /\left(5753269754265600 \pi^{9}\right) \\
&
\end{aligned}
$$

From (2), (6) we obtain our main results (1).

\section{Numerical Applications}

It is easy to check numerically (cf. [14, 16]) that the exact results obtained in the previous section are in agreement with the Airy function asymptotics. According to [15, 10, 14, the perturbative part of the partition function for $k=1$ is given by

\footnotetext{
${ }^{2}$ This result agrees perfectly with the numerical result for $N \leq 16$ in [16].
} 


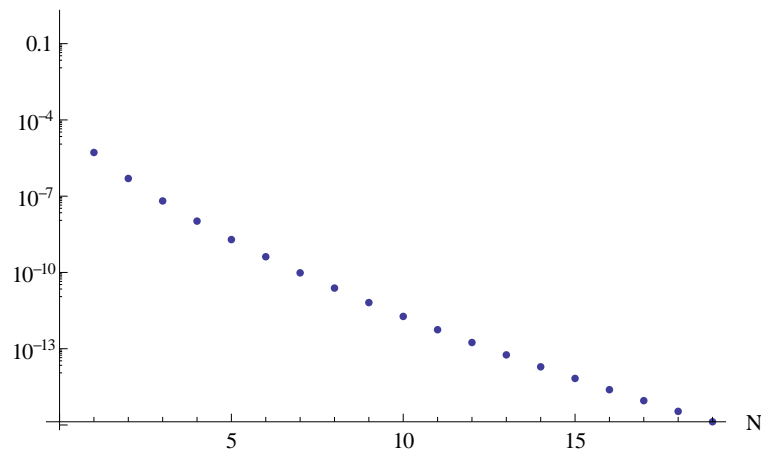

Figure 1: In this figure, the dots represent the sequence $Z(N) / Z^{(\text {pert })}(N)-1$.
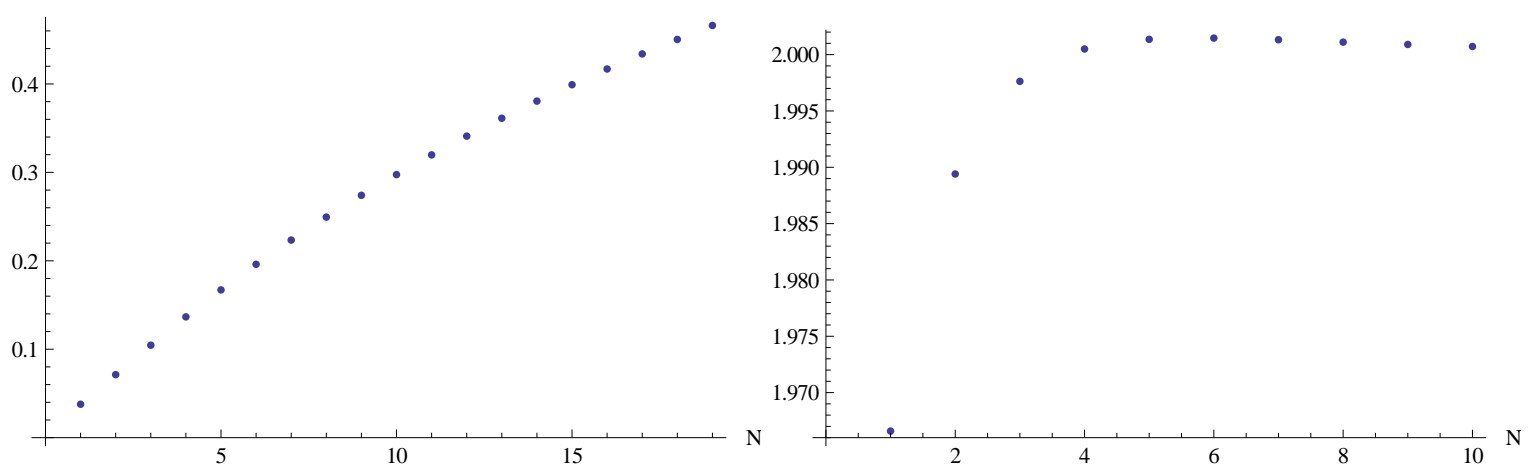

Figure 2: In these figures, the dots represent the sequence (24) (left) and its 9-th Richardson-like transform $\widetilde{F}^{(9)}(N)$ (right).

$$
Z^{\text {(pert) }}(N)=C e^{A} \operatorname{Ai}\left[C\left(N-\frac{1}{3}-\frac{1}{24}\right)\right]
$$

where

$$
\begin{gathered}
C=\frac{\pi^{2 / 3}}{2^{1 / 3}} \\
A=-\frac{\zeta(3)}{8 \pi^{2}}+\frac{1}{6} \log \frac{\pi}{2}+2 \zeta^{\prime}(-1)+\frac{1}{2} \log 2-\frac{1}{3} \int_{0}^{\infty} d x \frac{1}{e^{x}-1}\left(\frac{3}{x^{3}}-\frac{1}{x}-\frac{3}{x(\sinh x)^{2}}\right) .
\end{gathered}
$$

The Fig. 1 1 shows that indeed $Z(N)$ approaches $Z^{(\text {pert })}(N)$ exponentially fast. In [16] it was checked that the non-perturbative part $Z^{(\mathrm{np})} \equiv Z-Z^{\text {(pert) }}$ is suppressed by $e^{-2 \pi \sqrt{2 N}}$ which agrees with the previous analytical results $[6,10,9]$. One can go further and find the leading behavior of the prefactor. Namely, let us consider the following sequence:

$$
\widetilde{F}(N) \equiv \frac{Z^{(\mathrm{np})}(N)}{Z^{(\text {pert })}(N)} / N e^{-2 \pi \sqrt{2 N}}=\left(\frac{Z(N)}{Z^{(\text {pert })}(N)}-1\right) / N e^{-2 \pi \sqrt{2 N}} .
$$

From the previous works one expects that this sequence has an asymptotic expansion of the following form:

$$
\widetilde{F}(N)=c_{0}+\frac{c_{1}}{N^{1 / 2}}+\frac{c_{2}}{N}+\frac{c_{3}}{N^{3 / 2}}+\ldots
$$


This assumption will be verified numerically a posteriori. The graph of $\widetilde{F}(N)$ is shown on the left of Fig. 2. One can accelerate convergence of the sequence $\widetilde{F}(N)$ by performing Richardson-like transforms. Let us define the Richardson-like transform $\mathrm{R}_{\gamma}$ of a sequence $S(N)$ as

$$
\mathrm{R}_{\gamma}[S(N)] \equiv(N / \gamma+1) S(N+1)-N S(N) / \gamma
$$

Its crucial property is that

$$
\mathrm{R}_{\gamma}\left[c+O\left(\frac{1}{N^{\gamma}}\right)\right]=c+o\left(\frac{1}{N^{\gamma}}\right)
$$

In particular, if we define

$$
\widetilde{F}^{(n)}(N) \equiv \mathrm{R}_{\frac{n}{2}}\left[\widetilde{F}^{(n-1)}(N)\right] \quad(n \geq 1), \quad \widetilde{F}^{(0)}(N) \equiv \widetilde{F}(N),
$$

then one can show that

$$
\widetilde{F}^{(n)}(N)=c_{0}+O\left(\frac{1}{N^{\frac{n+1}{2}}}\right) .
$$

The graph of $\widetilde{F}^{(9)}(N)$ is shown on the right of Fig. 2. The sequence converges very fast, which verifies the self-consistency of the assumption (25). Our numerical result suggests that $c_{0}=2$ exactly. One can also numerically obtain $c_{1}, c_{2}, \ldots$ using similar techniques. On the M-theory side of the AdS/CFT correspondence this gives the 1-instanton contribution from M2-branes?

$$
\frac{Z^{(1-\text { inst })}}{Z^{\text {(pert) }}}=(2 N+O(\sqrt{N})) e^{-2 \pi \sqrt{2 N}} .
$$

It would be interesting to check this by a direct calculation of 1-instanton contribution in M-theory. Let us note that the prefactor in 30 cannot be obtained by previously developed techniques since they provide the non-perturbative part of the partition function as non-trivial asymptotic expansions either for large $k$ [6] or for small $k$ [10], whereas the result (30) is for $k=1$.

\section{Acknowledgments}

The authors would like to thank Aspen Center for Physics (NSF Grant No. 1066293) for hospitality. P. P. is supported by the Fonds National Suisse, subsidies 200020-126817 and 200020-137523, and by FASI RF 14.740.11.0347.

\section{References}

[1] A. Kapustin, B. Willett and I. Yaakov, JHEP 1003, 089 (2010) arXiv:0909.4559 [hep-th]].

[2] D. L. Jafferis, JHEP 1205, 159 (2012) arXiv:1012.3210 [hep-th]].

[3] N. Hama, K. Hosomichi, S. Lee, JHEP 1103, 127 (2011) arXiv:1012.3512 [hep-th]].

[4] I. R. Klebanov, A. A. Tseytlin, Nucl. Phys. B475, 164-178 (1996) hep-th/9604089.

[5] M. Mariño and P. Putrov, JHEP 1006, 011 (2010) [arXiv:0912.3074 [hep-th]].

\footnotetext{
${ }^{3}$ The smallest instanton action is twice the action of the D2-brane considered in 9 for $k=1$ because there is no M2 in $S^{7}$ which is a degree one cover of the D2-brane wrapping $\mathbb{R P}^{3} \in \mathbb{C P}^{3}$. However, there is an M2 wrapping $S^{3} \in S^{7}$ which is a degree two cover.
} 
[6] N. Drukker, M. Mariño, P. Putrov, Commun. Math. Phys. 306, 511-563 (2011) arXiv:1007.3837 [hep-th]].

[7] C. P. Herzog, I. R. Klebanov, S. S. Pufu, T. Tesileanu, Phys. Rev. D83, 046001 (2011) arXiv:1011.5487 [hep-th]].

[8] O. Aharony, O. Bergman, D. L. Jafferis and J. Maldacena, JHEP 0810, 091 (2008) arXiv:0806.1218 [hep-th]].

[9] N. Drukker, M. Mariño and P. Putrov, JHEP 1111, 141 (2011) arXiv:1103.4844 [hep-th]].

[10] M. Mariño and P. Putrov, J. Stat. Mech. 1203 (2012) P03001 arXiv:1110.4066 [hep-th]].

[11] A. B. Zamolodchikov, Nucl. Phys. B 432, 427 (1994) hep-th/9409108].

[12] C. A. Tracy and H. Widom, Commun. Math. Phys. 179, 667 (1996) solv-int/9509003.

[13] K. Okuyama, Prog. Theor. Phys. 127, 229 (2012) arXiv:1110.3555 [hep-th]].

[14] M. Hanada, M. Honda, Y. Honma, J. Nishimura, S. Shiba and Y. Yoshida, JHEP 1205, 121 (2012) arXiv:1202.5300 [hep-th]].

[15] H. Fuji, S. Hirano, S. Moriyama, JHEP 1108, 001 (2011) arXiv:1106.4631 [hep-th]].

[16] Y. Hatsuda, S. Moriyama and K. Okuyama, "Exact Results on the ABJM Fermi Gas," arXiv:1207.4283 [hep-th]. 